\title{
Implementation of Psychologically Informed Physical Therapy for Low Back Pain: Where Do We Stand, Where Do We Go?
}

\author{
Lindsay A Ballengee ${ }^{1-3}$ \\ Leah L Zullig ${ }^{3,4}$ \\ Steven Z George (iD) ${ }^{1,5}$ \\ 'Department of Orthopaedic Surgery, \\ Duke University, Durham, NC, USA; \\ ${ }^{2}$ Division of Physical Therapy, Duke \\ University, Durham, NC, USA; \\ ${ }^{3}$ Department of Population Health \\ Sciences, Duke University School of \\ Medicine, Durham, NC, USA; ${ }^{4}$ Center of \\ Innovation to Accelerate Discovery and \\ Practice Transformation, Durham \\ Veterans Affairs Health Care System, \\ Durham, NC, USA; ${ }^{5}$ Duke Clinical \\ Research Institute, Duke University, \\ Durham, NC, USA
}

\begin{abstract}
Low back pain continues to be a leading cause of disability and cost throughout the world. Evidence-based guidelines recommend use of non-pharmacological interventions to address decreases in physical function due to low back pain. Psychologically informed physical therapy (PIPT) is one way to effectively and efficiently address the need for nonpharmacological approaches. However, adoption of psychologically informed practice (PiP) by physical therapists has shown mixed results due to implementation challenges. In this perspective, we discuss the current state of PIPT training and implementation. We also propose a conceptual roadmap for future implementation needs related to increasing delivery of PIPT-informed approaches.
\end{abstract}

Keywords: implementation science, physical therapy, pain, non-pharmacological

\section{Introduction}

There has been substantial growth in interest in biopsychosocial treatments for patients with musculoskeletal pain. ${ }^{1}$ One reason for this increased interest is the American College of Physicians Clinical Practice Guidelines and the Centers for Disease Control and Prevention Guidelines for treatment of chronic pain., Additionally, the revised International Association for the Study of Pain (IASP) definition for pain focuses on the influence of biological, psychological, and social factors on a person's pain experience. ${ }^{4}$ This focus provides continued opportunities for non-pharmacological pain treatments that use psychological and behavioral components in the management of musculoskeletal pain.,

Physical therapists have incorporated psychologically informed practice (PiP) into their practice patterns for low back pain management. PiP is a novel approach that integrates physical, behavioral, and psychological interventions with an overall goal of reducing pain-related disability. ${ }^{5}$ Since PiP was originally proposed as a treatment framework; there has been an evolution and refinement in practice approaches for low back pain. PiP was targeted towards secondary prevention and subsequently focused treatment goals on reduction of disability. Recently, for low back pain, there has been a shift to focus more on whole person or positive health targets as patient outcomes. ${ }^{6}$

$\mathrm{PiP}$ is well positioned to be responsive to this shift away from secondary outcomes to a broader consideration of patient health. For example, key features of a PiP framework are well aligned with enhancing whole person health. These key
Correspondence: Lindsay A Ballengee 3II Trent Drive, Durham, NC, 277I0, USA

Tel + I 9194033702

Email lindsay.ballengee@duke.edu

Steven Z George 300 W. Morgan Street, Durham, NC, 2770I, USA

Tel + I 9196688300

Email steven.george@duke.edu 
features include addressing a patient's behavioral response to pain through any of the five major types of PiP interventions: 1) educational (threat reduction and activation; 2) behavioral (adapting behaviors in response to pain); 3) cognitive-behavioral (cognition and coping skills); 4) psychophysiological (stress reduction and mindfulness); and 5) contextual cognitive-behavioral (Acceptance and Commitment Therapy). ${ }^{7}$ The actual content of a PiP intervention varies and may best be thought of as a framework for therapies that integrate contextual, behavioral, and physical treatments. ${ }^{8}$ Psychologically informed physical therapy (PIPT) then is viewed as a subset of PiP when $\mathrm{PiP}$ is delivered by physical therapists. Therefore, in this paper when PiP is used it is a general reference to the treatment framework. When PIPT is used, it is referring to treatment delivery by physical therapists. Specifically, PIPT is an integration of traditional physical treatments (eg, spinal manipulation or exercise) with cognitivebehavioral treatments (eg, pain coping skills or progressive relaxation).

In this paper, we focus on PIPT. We fully acknowledge that other providers (eg, nurses, chiropractors, occupational therapists) have PiP models for delivery of treatments for chronic conditions. However, our implementation experiences primarily involve physical therapists, and we therefore provide insight based on our expertise. We acknowledge the important roles of other types of providers in PiP, but that is not the focus of this paper. PIPT can benefit patients by individualizing physical and psychological interventions to improve functional activities that had been limited due to pain. Although it is beyond the intended scope of this Perspective to exhaustively review PIPT, key treatment components include Realization, Relief, Regulation, Re-activation, and Reinforcement (5 R's). ${ }^{9}$ These core components are drawn from conceptual models that have been used in mental health and then applied in an integrated fashion (ie, with treatment of physical impairments) for pain clinic and rehabilitation settings. ${ }^{7,10,11}$ The conceptual models used in these clinical settings include the fear avoidance model, the ACT "hexaflex", and the cognitive behavioral model. ${ }^{12-14}$ This "5 R approach" to PIPT, including convergent examples for each of these conceptual models, are summarized in Table 1.

There is growing evidence demonstrating the effectiveness of PIPT approaches for pain-related conditions. Early supporting evidence came from the United Kingdom, where a stratified care approach was investigated for managing patients with low back pain in a randomized controlled trial. ${ }^{15}$ Low-risk patients were given minimal treatment, medium risk patients were referred for traditional physical therapy, and high-risk patients received psychologically informed physical therapy. Results showed that the stratified care intervention group had favorable outcomes for disability scores, health quality of life, and cost savings. A trial by Beneciuk and George ${ }^{16}$ reported on the pragmatic application of risk stratification in outpatient physical therapy practices and found that physical therapists who were randomly selected to receive stratified care training had increased biopsychosocial treatment orientations and patients who received care from these clinicians had improved pain and disability scores. These trials demonstrated that physical therapists can effectively screen and deliver psychologically informed care which resulted in better patient outcomes.

Evidence from individual randomized controlled trials has been reported for synthesis of PIPT approaches for musculoskeletal pain. For example, Hall et al conducted a systematic review that assessed the effectiveness of physical therapist-delivered cognitive behavioral interventions for low back pain. ${ }^{17}$ Five randomized controlled trials $(\mathrm{n}=1390)$ were identified and meta-analysis revealed that cognitive-behavioral interventions had a greater effect on reduction of disability and pain than education or exercise interventions. ${ }^{17}$ Similarly, Archer et al conducted another review that investigated the use of psychologically informed physical therapy for different types of musculoskeletal pain and identified eight randomized controlled trials $(n=978) .{ }^{18}$ They found that PIPT is likely effective for low back pain, especially when it is targeted toward patients who are at high risk of poor outcomes due to fear of movement. ${ }^{18}$ Two years later, Coronado et al identified 18 randomized controlled trials $(\mathrm{n}=2387)$ that used PIPT approaches for patients with musculoskeletal pain and found that PiP interventions had a greater effect on reduction of disability and pain than education or exercise-only interventions. ${ }^{17,19}$

A notable observation for this evidence base is that explanatory clinical trials investigating PIPT for low back pain have a tendency to show favorable results; however, pragmatic trials have shown less promising outcomes. For example, the TARGET trial (77 primary care clinics, $\mathrm{n}=$ 2300 enrolled patients) investigated whether a riskstratified approach would result in lower rates of chronic low back pain and improved self-reported disability. Patients in the high-risk group were referred to PIPT or 
Table I Key Components Comprising Psychologically Informed Practice (PIPT)

\begin{tabular}{|c|c|c|}
\hline \multicolumn{3}{|c|}{ Over-Arching Components of PIPT } \\
\hline & Rationale/Goals/Objectives & $\begin{array}{l}\text { Concrete } \\
\text { Example }\end{array}$ \\
\hline $\begin{array}{l}\text { Patient Centered } \\
\text { Communication }\end{array}$ & $\begin{array}{l}\text { Realization - shared } \\
\text { understanding } \\
\text { Understanding the pain } \\
\text { experience by actively eliciting } \\
\text { patient perspective, including } \\
\text { but not limited to psychosocial } \\
\text { and cultural contexts }\end{array}$ & $\begin{array}{l}\text { Motivational } \\
\text { Interviewing } \\
\text { Shared } \\
\text { Decision } \\
\text { Making }\end{array}$ \\
\hline \multicolumn{3}{|c|}{ Treatment Specific Components } \\
\hline & Rationale/Goals/Objectives & $\begin{array}{l}\text { Concrete } \\
\text { Example }\end{array}$ \\
\hline Pain Modulation & $\begin{array}{l}\text { Relief - pain intensity and } \\
\text { severity } \\
\text { Short-term primarily aimed at } \\
\text { decreasing intensity/severity, } \\
\text { can be delivered by provider } \\
\text { but should also include self- } \\
\text { management components }\end{array}$ & $\begin{array}{l}\text { TENS } \\
\text { Manual } \\
\text { Therapy } \\
\text { Physical } \\
\text { Modalities } \\
\text { Directional } \\
\text { Preference } \\
\text { Exercise }\end{array}$ \\
\hline
\end{tabular}

Reinforcement $=$ providing encouragement when patient reports using hot packs at home to help relieve pain.

\begin{tabular}{|l|l|l|}
\hline Pain Coping Skills & $\begin{array}{l}\text { Regulation - physical and } \\
\text { emotional } \\
\text { Pain relief is not a direct target } \\
\text { but may be a "side effect" of } \\
\text { altering the individual's sensory } \\
\text { environment to allow for } \\
\text { different processing of } \\
\text { nociception. }\end{array}$ & $\begin{array}{l}\text { Progressive } \\
\text { Muscle }\end{array}$ \\
& Relaxation \\
Activity \\
pacing \\
Education \\
Mindfulness
\end{tabular}

Reinforcement $=$ answering questions and practicing deep breathing exercises in clinic

\begin{tabular}{|c|c|c|}
\hline $\begin{array}{l}\text { Activity and } \\
\text { Exercise } \\
\text { Recommendations }\end{array}$ & $\begin{array}{l}\text { Re-activation - physical } \\
\text { activity About preventing } \\
\text { disability through avoidance, } \\
\text { encourage resumption of } \\
\text { activities despite the pain, and } \\
\text { behavioral/psychological dosing } \\
\text { principles }\end{array}$ & $\begin{array}{l}\text { Graded } \\
\text { Exposure } \\
\text { Graded } \\
\text { Activity }\end{array}$ \\
\hline
\end{tabular}

Reinforcement $=$ praising patient for reaching activity goal in clinic, despite pain levels not improving yet

usual care. At the conclusion of the trial, patients in the PIPT intervention group showed no differences in disability, care utilization, or transition to chronic low back pain. $^{20}$

An earlier study, the MATCH trial (6 primary care clinics, $n=603$ enrolled patients), investigated whether use of the STarT Back risk-stratification strategy for patients with low back pain resulted in superior outcomes for physical function and pain severity. ${ }^{21}$ While clinicians used the STarT Back tool with half of participants, use of the tool did not change recommended treatments nor did the intervention have any significant effects on patient outcomes. $^{21}$

Common themes for both the TARGET and MATCH trials are that they were conducted in the United States and reported implementation challenges for PIPT approaches. In TARGET, the authors report a significant "implementation shortfall" with the PIPT referral process resulting in only half of the patients in the stratified care group receiving a PIPT referral. ${ }^{20}$ This implementation shortfall was attributed to electronic health record challenges with the referral process to PIPT clinicians, as well as a lack of study-funded resources for physical therapy visits. ${ }^{20}$ Similarly, in MATCH, the authors discuss implementation difficulties due to the complexity of the PIPT intervention, as well as the nuances of the US healthcare system, when compared to the U.K. ${ }^{21}$

Accordingly, this perspective addresses issues of PIPT implementation within US health systems. The fact that the TARGET and MATCH trials had challenges implementing this type of intervention in a pragmatic trial is concerning for those interested in implementing PIPT in real-world clinical settings. One challenge limiting uptake of PIPT in care settings is that, in general, nonpharmacological interventions can be challenging for health systems to deliver, when compared to medications or surgery. ${ }^{1,22}$ Clinician attitudes and beliefs about using behavioral and psychological interventions can also influence their willingness to use PiP approaches since they are still not commonly taught in pre-professional training for many provider types (eg, physical therapists). ${ }^{23,24}$ Other challenges to PIPT implementation include physical therapists feeling unprepared to treat patients with complex pain conditions and a fear of not meeting patient's expectations for carrying out traditional physical therapy care. ${ }^{25}$

Comprehensive training in implementing PiP approaches for physical therapists would address some of the challenges of real-world adoption; however, most PIPT training has been completed in support of conducting clinical trials. Scant evidence exists on how to scale PIPT training so that 
it can be delivered for practicing physical therapists. ${ }^{19}$ For example, the ideal training mode, intensity, or duration for learning and implementing PIPT interventions within realworld clinical settings is unknown. Indeed, the recent clinical trial experiences from the US provide an indication that scaling PIPT to meet the demands of routine clinical delivery is primarily implementation related. ${ }^{20,21}$

Therefore, the purpose of this Perspective is twofold. First, we discuss the current state of PIPT training and implementation. Second, we propose a roadmap for future implementation needs related to increasing delivery of PiPinformed approaches by physical therapists. Within this perspective, we have organized these into two concepts: Concept 1 - Current state of PIPT implementation and Concept 2 - Describe an implementation roadmap for PIPT.

\section{Concept I: Current State of PIPT Implementation}

Most of the implementation of PiP approaches for physical therapists has been done in preparation for conducting clinical trials. In these instances, physical therapists are trained in a specific PiP intervention (eg, Pain Coping Skills Training, ${ }^{26}$ Acceptance and Commitment Therapy ${ }^{27}$ ) and use those skills to treat trial participants with a given pain diagnosis. These trainings consist of different combinations of didactic, experiential, and mentoring components. Examples of how PIPT trainings have been delivered for selected clinical trials are shown in Table 2. In the selected trials, training episodes were between one and six days and included didactic and experiential learning. Three of the five PIPT trainings also had ongoing mentoring and feedback components. Two manualized trials for PIPT also used fidelity checklists to assess physical therapist competence and adherence.

One advantage of training for explanatory trials is the ability to focus on treatment fidelity through many different means, including protocol standardization and a dedicated provider pool. If PIPT interventions do not demonstrate positive effects in such highly controlled environments, then they are not likely to be effective in an environment with fewer resources and less time dedicated towards delivering the intervention. However, there are also disadvantages with this method of training. Explanatory trials can take a long time to complete and are expensive. Typically, clinicians who are trained in PIPT interventions are paid research staff. After trial completion, the clinic or health system no longer has access to those specially trained providers unless they have the resources to hire additional personnel.

Embedded pragmatic trials are one potential solution to the sustainability concerns of an explanatory trial. In an embedded pragmatic trial, clinicians who are integrated within an existing clinic or health system are trained to deliver the PIPT interventions used in the study protocol. This use of existing clinical resources can be valuable for increasing the likelihood of long-term sustainability of the PIPT interventions. Additionally, like PIPT training for explanatory trials, training for pragmatic trials is highly standardized. However, variation from the treatment protocol is more acceptable (and even expected) due to the practical focus.

There is convergence around core components of a PIPT training program (ie, didactic content, experiential learning, and mentoring with feedback) as shown by the different training protocols from Table 2. However, there is no consensus on how these training programs should be optimally delivered. For example, we do not know the most effective intensity of training delivery or how to increase uptake of PIPT practice into an existing health system. This lack of guidance for implementation may help to explain the small treatment effects of PIPT interventions in recent trials that were more pragmatic in nature and could be attributed to undertraining or not appropriately stratifying care. ${ }^{19,28}$ Further research into the implementation of PIPT training could elucidate effective combinations of training modes and implementation strategies within specific contexts (academic medical center, private clinic, home health, etc.), as well as for specific patient populations.

Table 2 indicates where PIPT implementation has been focused to-date. However, as clinical trial results have been published, it is clear that integrating PIPT approaches into the US healthcare system is not a simple task..$^{20,21}$ The strategies that have been used to implement PIPT approaches for clinical trials in other countries have not generalized to effective real-world delivery for trials embedded in the US healthcare system. This issue may not be specific to PIPT, as other management approaches for low back pain have struggled with real-world adoption. ${ }^{29,30}$

\section{Concept 2: Road Map for PIPT Implementation}

One way to make the leap toward real-world delivery of behavioral interventions has been to move from clinical trials to implementation-focused studies. ${ }^{31-33}$ Brown 
Table 2 PIPT Training Examples for Licensed Physical Therapists

\begin{tabular}{|c|c|c|c|c|}
\hline $\begin{array}{l}\text { Research Physical Therapists in } \\
\text { TARGET Trial }{ }^{59}\end{array}$ & $\begin{array}{l}\text { Research Physical } \\
\text { Therapists in Knee OA } \\
\mathbf{R C T}^{26}\end{array}$ & $\begin{array}{l}\text { Outpatient MSK } \\
\text { Physical Therapists }^{27}\end{array}$ & $\begin{array}{l}\text { Primary Care Clinicians } \\
\text { and Outpatient Physical } \\
\text { Therapists in MATCH } \\
\text { Trial }^{21}\end{array}$ & $\begin{array}{l}\text { Outpatient } \\
\text { Physical } \\
\text { Therapists in } \\
\text { STarT Back } \\
\text { Trial }^{15}\end{array}$ \\
\hline One day course, primarily didactic & $\begin{array}{l}\text { Ten, } 45 \text {-min modules } \\
\text { delivered over } 4 \text { days with } \\
\text { ongoing mentoring/ } \\
\text { feedback }\end{array}$ & $\begin{array}{l}\text { Two-day course, mixture } \\
\text { of didactic and } \\
\text { experiential, training } \\
\text { manual, monthly } \\
\text { supervision/feedback }\end{array}$ & $\begin{array}{l}\text { Six didactic sessions for } \\
\text { primary care, five days of } \\
\text { intensive training for PTs } \\
\text { with ongoing mentoring and } \\
\text { supervision }\end{array}$ & $\begin{array}{l}\text { One-to-six-day } \\
\text { training } \\
\text { depending on } \\
\text { intervention } \\
\text { group }\end{array}$ \\
\hline $\begin{array}{l}\text {-Pain Attitudes and Beliefs Scale for } \\
\text { Physical Therapists } \\
\text { - Confidence in PIPT skill application }\end{array}$ & $\begin{array}{l}\text {-Treatment fidelity } \\
\text { checklist to measure } \\
\text { therapist adherence to } \\
\text { protocol/manual and } \\
\text { session performance }\end{array}$ & $\begin{array}{l}\text {-Treatment fidelity } \\
\text { checklist to measure } \\
\text { therapist adherence to } \\
\text { protocol/manual and } \\
\text { session performance }\end{array}$ & $\begin{array}{l}\text {-Electronic healthcare record } \\
\text { monitoring }\end{array}$ & $\begin{array}{l}\cdot \text { Health record } \\
\text { auditing }\end{array}$ \\
\hline $\begin{array}{l}\text { I. Summarize relationships between } \\
\text { pain neuroscience, pain models, and } \\
\text { the development and maintenance } \\
\text { of chronic low back pain } \\
\text { 2. Identify patients at high risk for } \\
\text { transitioning from acute to chronic } \\
\text { low back pain } \\
\text { 3. Apply targeted treatment for } \\
\text { patients at high risk for transitioning } \\
\text { from acute to chronic low back pain } \\
\text { 4. Understand primary assumptions } \\
\text { of CBT and specific skills associated } \\
\text { with CBT based interventions } \\
\text { 5. Recognize effective } \\
\text { communication skills and be able to } \\
\text { implement as a key component to } \\
\text { PIPT }\end{array}$ & $\begin{array}{l}\text { I. Establishes/maintains } \\
\text { rapport } \\
\text { 2. Applies program } \\
\text { protocol to participants' } \\
\text { situation/challenges } \\
\text { 3. Encourages participant } \\
\text { involvement in the } \\
\text { sessions } \\
\text { 4. Asks open-ended } \\
\text { questions, encourages } \\
\text { change, rolls with } \\
\text { resistance, supports self- } \\
\text { efficacy }\end{array}$ & Not reported & Not reported & Not reported. \\
\hline
\end{tabular}

et al have created a dissemination and implementation diagram to increase real-world relevance and adoption of evidence-based interventions (Figure 1). ${ }^{34}$ The preintervention phase often consists of theory development and pilot studies to investigate if an intervention is feasible to carry out. For PIPT training approaches, there are examples available to guide recommendations for the future.

For pre-licensure physical therapists, Ballengee et al have proposed a training approach that is similar to the one used for practicing clinicians with an additional focus on foundational pain education and patient-centered communication. $^{35}$ This training method addresses the call for pre-licensure training to go beyond didactic content and provide more experiential training to address barriers with incorporating psychologically informed interventions. $^{36,37}$ The training focuses on addressing the complex nuances of behavioral-based nonpharmacologic pain treatments like building therapeutic alliance, reducing the perceived threat of pain, conceptualizing pain beliefs, and promoting self-efficacy. ${ }^{38}$ Prelicensure trainings like this one are being introduced throughout Doctor of Physical Therapy curriculums throughout the U.S. ${ }^{39}$ One noteworthy limitation of addressing PIPT implementation challenges through this type of training is the potential lack of reinforcement upon graduation if the PT is not working in an environment with PIPT-specific mentoring. 


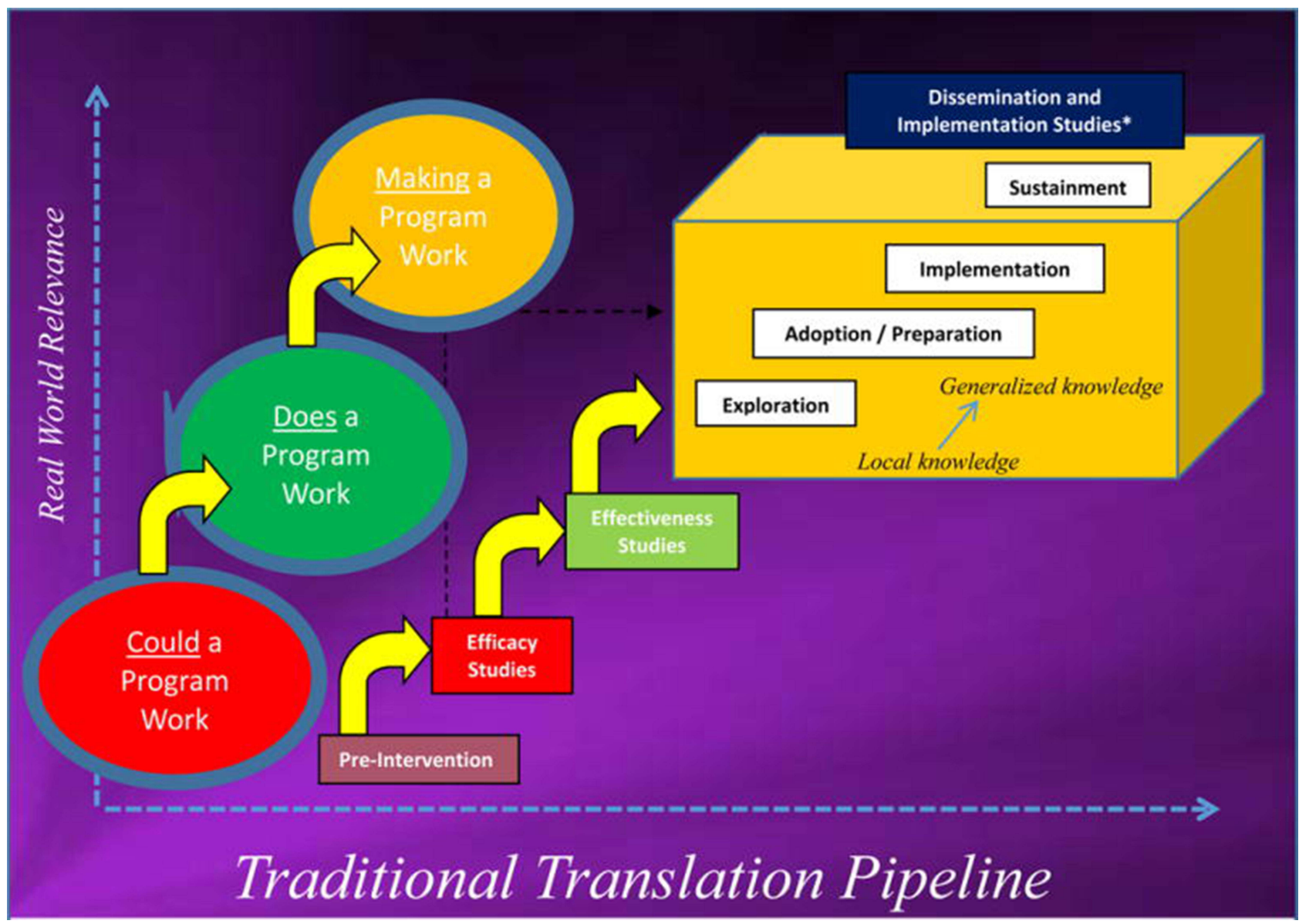

Figure I Dissemination and implementation framework.

Notes: Reprinted from Brown CH, Curran G, Palinkas LA et al. An Overview of Research and Evaluation Designs for Dissemination and Implementation. Annu Rev Public Health. 2017;38:I-22. Copyright (C) 2017 Annual Reviews. This work is licensed under a Creative Commons Attribution-ShareAlike 4.0 (CC-BY-SA). ${ }^{34}$

Examples of real-world adoption of PIPT training for licensed physical therapists have been shown in the United States. Keefe et al have developed a PIPT training program for practicing physical therapists that consists of a PIPT treatment manual, experiential workshops, and ongoing supervision with consultation and feedback. ${ }^{7}$ This multicomponent training approach gives clinicians the opportunity to build their knowledge and PIPT skills while also addressing treatment fidelity. This approach has been carried out within the Duke University Health Care System with 95 clinicians (80 physical therapists, 15 occupational therapists) being trained to-date. The program works with a group of 35 clinicians at a time and consists of 90 minute didactic and experiential sessions for 15 weeks with a pain psychologist. This has been implemented as a quality improvement initiative funded by the hospital system and outcomes are currently being evaluated on a clinician and system-wide level.
Another quality improvement initiative is taking place within the Department of Veteran's Affairs Health System (VA) to train physical therapists in PiP approaches. This approach uses physical therapists who have already been trained in PIPT interventions to train other VA physical therapists (train-the-trainer approach) who are interested in learning components of the International Association for the Study of Pain (IASP) pain education curriculum. ${ }^{40}$ The VA curriculum consists of didactic, experiential, and mentoring components. This quality improvement project will also evaluate the implementation strategies needed for widescale adoption after two pilot clinics go through the training. This initiative coincides with the American Physical Therapy Association's (APTA) call to standardize pain education and advance therapeutic pain communication for physical therapists. ${ }^{41}$ Notably, both Duke University Health System and the Department of Veterans Affairs are well-resourced settings that may not be indicative of all practice settings across the United States. 


\section{Moving Towards Wide-Scale Implementation}

While there are some examples of local attempts to implement PIPT approaches, there is no over-arching guidance on the most effective way to increase real-world clinical delivery. Figure 2 illustrates a conceptual PIPT Implementation Roadmap adapted from the Brown et al diagram that could bridge the gap between training for trials and real-world adoption by focusing on "Implementation Zones" between the different stages. ${ }^{34}$ To-date, no implementation-focused studies like those suggested in Figure 2 have been done for PIPT interventions. One way to conceptualize implementation studies for PIPT would be to consider different types of research questions that would align with the Implementation Zones from Figure 2. Curran et al proposed three hybrid effectivenessimplementation study designs that could serve as a staged approach to move PIPT beyond pure efficacy trials. ${ }^{42,43}$ Table 3 illustrates the three hybrid designs with corresponding PIPT-focused aims and research questions.

\section{Determining PIPT Effectiveness and Approaches for Implementation}

An example of a Type 1 hybrid design for a PIPT study would be to investigate whether a PIPT approach resulted in improved functional outcomes for patients with low back pain in an outpatient physical therapy clinic. While the focus of a Type 1 study is on PIPT effectiveness, the study investigators would also gather information about barriers and facilitators to implementing the PIPT approaches to inform future studies. In a Type 2 hybrid design, the focus of the study would be on both the effectiveness of the PIPT approach on functional outcomes for low back pain patients, and on the feasibility of using a particular implementation intervention or strategy (eg, in-person, PIPT training). The unit of randomization in a Type 1 study would likely be the patient, while in a Type 2 study, patients would often be randomized for the clinical effectiveness aim and the provider or clinical unit would often be the unit of randomization for the implementation aim.

\section{Determining Best Approach to Implement PIPT}

In a Type 3 implementation study, researchers could investigate whether one implementation strategy is superior to another based on a pre-determined set of implementation outcomes. An example of a Type 3 PIPT implementation study could be to start with an existing PIPT curriculum that was been used for training clinicians in a pragmatic trial. To study the most effective way of implementing that

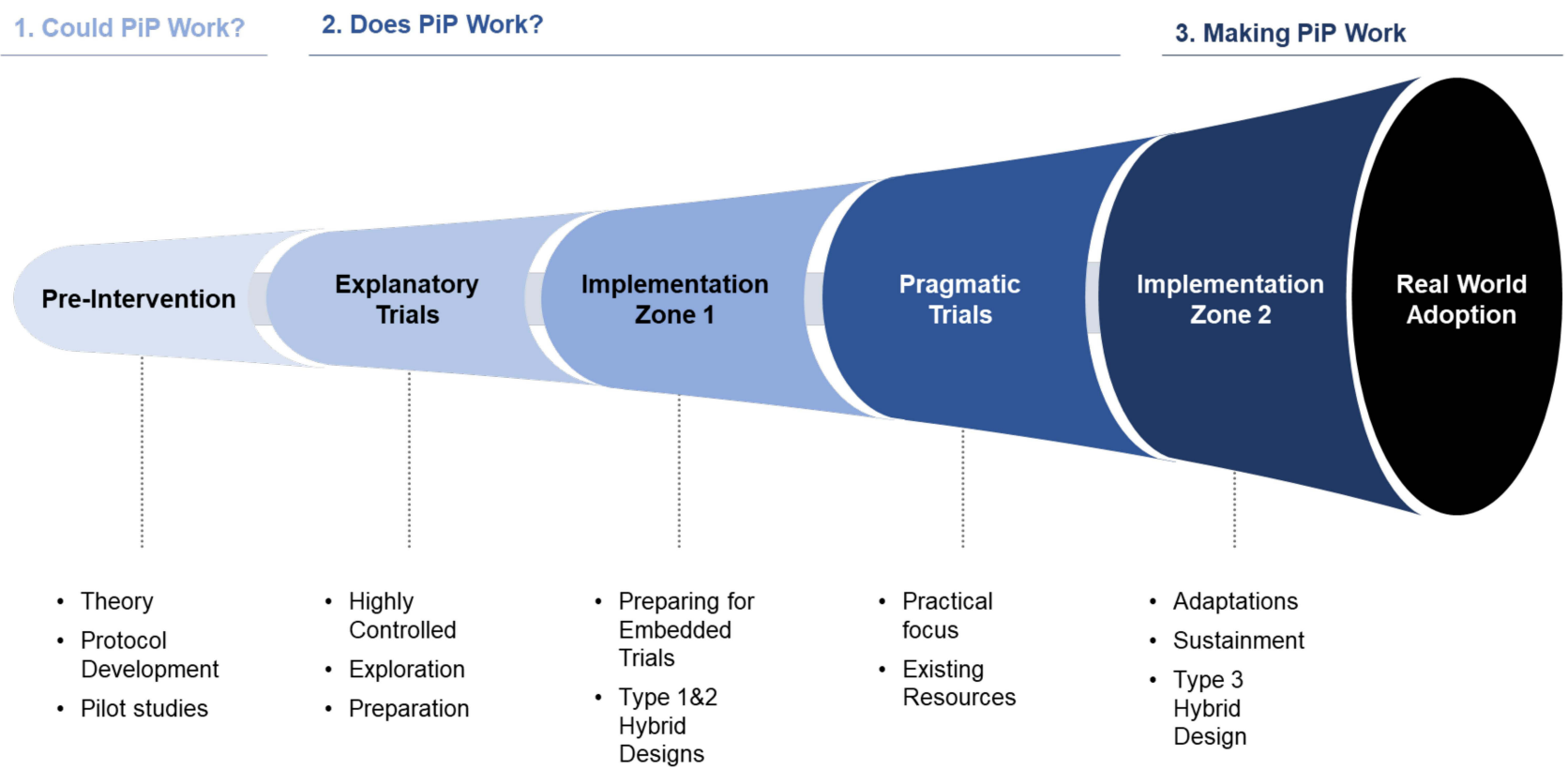

Figure 2 PIPT implementation roadmap. 
Table 3 Hybrid Effectiveness-Implementation Study Designs with Corresponding PIPT Research Questions, Clinical Outcomes, and Implementation Outcomes ${ }^{43}$

\begin{tabular}{|c|c|c|}
\hline Type I & Type 2 & Type 3 \\
\hline $\begin{array}{l}\text { Focus: Effectiveness of intervention with } \\
\text { "exploration" of intervention implementation }\end{array}$ & $\begin{array}{l}\text { Focus: Dual focus on effectiveness and } \\
\text { implementation outcomes }\end{array}$ & Focus: Implementation outcomes \\
\hline $\begin{array}{l}\text { Primary aim: Determine effectiveness of PiP } \\
\text { approach for patients with low back pain. } \\
\text { Secondary aim: Better understand context for } \\
\text { using PiP approaches in outpatient physical } \\
\text { therapy settings. } \\
\text { Primary question: Does a one-day PiP training } \\
\text { program for outpatient physical therapists } \\
\text { result in improved functional outcomes for } \\
\text { patients with low back pain? } \\
\text { Secondary question: What are the barriers and } \\
\text { facilitators for using PiP approaches for } \\
\text { patients with low back pain? }\end{array}$ & $\begin{array}{l}\text { Co-primary aim: Determine effectiveness of } \\
\text { PiP approach while also determining feasibility } \\
\text { and utility of a particular implementation } \\
\text { intervention or strategy. } \\
\text { Co-primary questions: Does a PiP approach } \\
\text { work for patients with low back pain in an } \\
\text { outpatient physical therapy clinic? Does the } \\
\text { implementation method (one-day, in-person } \\
\text { PiP training program) show promise in } \\
\text { facilitating use of PiP approaches in this } \\
\text { context? }\end{array}$ & $\begin{array}{l}\text { Primary aim: Determine utility of a specific } \\
\text { implementation intervention or strategy (eg } \\
\text { one-day, in-person PiP training program vs } \\
\text { online PiP training module). } \\
\text { Secondary aim: Assess functional outcomes for } \\
\text { patients with low back pain. } \\
\text { Primary question: Which implementation } \\
\text { method (in-person training vs online module) } \\
\text { works better at facilitating use of PiP } \\
\text { approaches in clinic? } \\
\text { Secondary questions: Are functional outcomes } \\
\text { acceptable? Are the changes in pain scores } \\
\text { clinically meaningful? What are the utilization } \\
\text { rates of PiP approaches for in-person training } \\
\text { vs online training? }\end{array}$ \\
\hline
\end{tabular}

PIPT curriculum within a health system, a Type 3 study could be conducted to compare an audit and feedback strategy against academic detailing and evaluate which strategy produced superior implementation outcomes. ${ }^{44}$

Another Type 3 option might be to investigate implementing PIPT interventions in a stepped-wedge design where all sites receive a low-level implementation strategy such as a one-day course with a manual (academic detailing) and then evaluate where those sites are with their implementation outcomes at a given time point. Those sites that have not made sufficient progress would then be "stepped-up" to receive a higher level (ie, more resource-intensive) implementation strategy such as implementation facilitation by a trained facilitator. ${ }^{44}$

\section{Other Issues That Could Impact PIPT Implementation}

Barriers to PIPT implementation should be considered at multiple levels (eg, individual, clinic-level, health-system, and beyond). ${ }^{4,46}$ For example, in pragmatic trials in the US, a lack of primary care referrals have been observed as a barrier to patients receiving PIPT. Several of the challenges with PIPT implementation (ie, no psychosocial risk screening, lack of PT referral) start at the primary care visit where many patients do not have the opportunity to access psychologically informed care. ${ }^{47-49}$ Instituting routine screening in primary care settings with automatic referral to PIPT could limit this barrier. However, such a process could create other problems (ie, not enough providers to deliver treatment, increase risk of overtreatment). Future research should investigate the impact of different referral models on the percentage of those actually receiving PIPT.

Another example of limiting PIPT implementation is existing policies that limit reimbursement for nonpharmacological pain treatments. Therefore, policyrelated changes could be used to increase reimbursement for non-pharmacological management of low back pain. ${ }^{50}$ However, the upfront resource investment (eg, time, money, personnel) required to implement nonpharmacological treatments, may be too much for a health system if there are no reimbursement incentives in place to offset those costs. One solution may be to conduct implementation-focused studies that carefully consider context, including current clinical workflow, to optimize delivery approaches, elucidate the most effective ways to implement PIPT in a given setting, and therefore optimize resource use - all with the goal of providing the best clinical care with the highest return on investment.

Another policy-related change could be to alter the way funding can be used for a trial. Often, funding policies are geared towards the start-up and conduct of the trial. These funds are used in study start-up for 
confirming the protocol, hiring study staff, completing training for intervention delivery, reimbursing participants, and setting up systems to collect outcome measures. During the conduct of the trial, funds are used to deliver the intervention, collect outcome measures, complete analyses to determine intervention effectiveness, and to disseminate trial findings. Typically, there are not sufficient funds after trial completion that would add to the sustainability of the intervention in the setting in which the trial was conducted. This type of funding would be especially appealing for interventions that demonstrate effectiveness during the trial, but it would require different funding mechanisms than are currently available.

Lastly, another important consideration for implementing PIPT approaches is context, or the set of unique circumstances and factors around implementation of PIPT (eg, staffing availability, culture around PT's role, etc.). For example, each health-care setting has unique characteristics that could impact the success or failure of an implementation effort. $^{51,52}$ For non-pharmacologic approaches like PIPT, part of the contextual considerations that have impacted its uptake include provider beliefs. ${ }^{1}$ On the positive side, physical therapists are known for their patient-centered approaches to care through the use of tools like shareddecision making and patient-centered communication. ${ }^{53,54}$ However, physical therapy training is still heavily focused on biomedical approaches for treating musculoskeletal pain which could make adoption of PIPT more difficult. ${ }^{39}$ Practicing physical therapists often have to self-teach or selffinance continuing education courses to learn about PiP approaches. Other factors related to physical therapist role clarity have been cited as potential barriers. ${ }^{55}$ Some physical therapists report difficulty in getting buy-in from patients when using PiP approaches due to the perception that physical therapists only focus on physical activity and exercise. ${ }^{56}$

\section{Conclusion}

Pain care in the United States has many improvements to make towards systematic implementation of effective, evidence-based, nonpharmacological treatments. ${ }^{57,58} \mathrm{PiP}$ delivered by physical therapists is one way to address this need, but while there is evidence to support its effectiveness for certain patient populations, evidence is lacking about the best way to implement these interventions into everyday clinical care. In this Perspective, we have presented a conceptual roadmap to move implementation of PIPT beyond the delivery of clinical trials and towards real- world adoption. This roadmap serves as guidance to increasing PIPT scalability and scope, which if fully realized could help improve routine delivery of guideline concordant pain care. Physical therapists can be the provider of choice for delivering PiP approaches, but only if the scale of training can be increased to meet existing needs.

\section{Disclosure}

Dr Leah L Zullig reports grants from Proteus Digital Health and the PhRMA Foundation awarded to her institution, as well as other support from Novartis and Pfizer. All of this funding is outside the submitted work. Dr Steven $\mathrm{Z}$ George reports grants from NIH, personal fees from Rehab Essentials, Inc, and personal fees from Med Risk, LLC; all outside the submitted work. He is on the Editorial Board for the following journals: Physical Therapy, Journal of Pain, and Archives of Physical Medicine \& Rehabilitation. The authors report no other conflicts of interest in this work.

\section{References}

1. George SZ, Lentz TA, Goertz CM. Back and neck pain: in support of routine delivery of non-pharmacologic treatments as a way to improve individual and population health. Transl Res. 2021. doi:10.1016/j.trs1.2021.04.006

2. Qaseem A, Wilt TJ, McLean RM, Forciea MA; Clinical Guidelines Committee of the American College of Physicians. Noninvasive treatments for acute, subacute, and chronic low back pain: a clinical practice guideline from the American College of Physicians. Ann Intern Med. 2017;166(7):514-530. doi:10.7326/M16-2367

3. CDC guideline for prescribing opioids for chronic pain-United States, 2016 | Guidelines | JAMA. JAMA Network. Available from: https://jamanetwork-com.proxy.lib.duke.edu/journals/jama/articleabstract/2503508. Accessed November 13, 2019.

4. IASP announces revised definition of pain. ISAP. Available from: https://www.iasp-pain.org/PublicationsNews/NewsDetail.aspx? ItemNumber=10475. Accessed December 7, 2020.

5. Main CJ, George SZ. Psychologically informed practice for management of low back pain: future directions in practice and research. Phys Ther. 2011;91(5):820-824. doi:10.2522/ptj.20110060

6. Buchbinder R, van Tulder M, Öberg B, et al. Low back pain: a call for action. Lancet. 2018;391(10137):2384-2388. doi:10.1016/S01406736(18)30488-4

7. Keefe FJ, Main CJ, George SZ. Advancing psychologically informed practice for patients with persistent musculoskeletal pain: promise, pitfalls, and solutions. Phys Ther. 2018;98(5):398-407. doi:10.1093/ ptj/pzy024

8. Nicholas MK, George SZ. Psychologically informed interventions for low back pain: an update for physical therapists. Phys Ther. 2011;91 (5):765-776. doi:10.2522/ptj.20100278

9. Ballengee LA, George SZ. Towards a better understanding of psychologically informed practice for physical therapists. In: Lifestyle Medicine for the Physical Therapist. In press

10. Fordyce WE, Fowler RS, Lehmann JF, Delateur BJ, Sand PL, Trieschmann RB. Operant conditioning in the treatment of chronic pain. Arch Phys Med Rehabil. 1973;54(9):399-408. 
11. Keefe FJ, Rumble ME, Scipio CD, Giordano LA, Perri LM. Psychological aspects of persistent pain: current state of the science. J Pain. 2004;5(4):195-211. doi:10.1016/j.jpain.2004.02.576

12. Vlaeyen JWS, Kole-Snijders AMJ, Boeren RGB, van Eek H. Fear of movement/(re)injury in chronic low back pain and its relation to behavioral performance. Pain. 1995;62(3):363-372. doi:10.1016/ 0304-3959(94)00279-N

13. McHugh L. A new approach in psychotherapy: ACT (acceptance and commitment therapy). World J Biol Psychiatry. 2011;12(sup1):76-79. doi:10.3109/15622975.2011.603225

14. Beck JS. Cognitive Therapy. In: The Corsini Encyclopedia of Psychology. American Cancer Society; 2010:1-3. doi:10.1002/ 9780470479216.corpsy0198

15. Hill JC, Whitehurst DG, Lewis M, et al. Comparison of stratified primary care management for low back pain with current best practice (STarT Back): a randomised controlled trial. Lancet. 2011;378 (9802):1560-1571. doi:10.1016/S0140-6736(11)60937-9

16. Beneciuk JM, George SZ. Pragmatic implementation of a stratified primary care model for low back pain management in outpatient physical therapy settings: two-phase, sequential preliminary study. Phys Ther. 2015;95(8):1120-1134. doi:10.2522/ptj.20140418

17. Hall A, Richmond H, Copsey B, et al. Physiotherapist-delivered cognitive-behavioural interventions are effective for low back pain, but can they be replicated in clinical practice? A systematic review. Disabil Rehabil. 2018;40(1):1-9. doi:10.1080/09638288.2016.1236155

18. Archer KR, Coronado RA, Wegener ST. The role of psychologically informed physical therapy for musculoskeletal pain. Curr Phys Med Rehabil Rep. 2018;6(1):15-25. doi:10.1007/s40141-018-0169-x

19. Coronado RA, Brintz CE, McKernan LC, et al. Psychologically informed physical therapy for musculoskeletal pain: current approaches, implications, and future directions from recent randomized trials. Pain Rep. 2020;5(5):e847. doi:10.1097/PR9.0000000000000847

20. Delitto A, Patterson CG, Stevans JM, et al. Stratified care to prevent chronic low back pain in high-risk patients: the TARGET trial. A multi-site pragmatic cluster randomized trial. EClinicalMedicine. 2021;34:100795. doi:10.1016/j.eclinm.2021.100795

21. Cherkin D, Balderson B, Wellman R, et al. Effect of low back pain risk-stratification strategy on patient outcomes and care processes: the MATCH randomized trial in primary care. $J$ Gen Intern Med. 2018;33(8):1324-1336. doi:10.1007/s11606-018-4468-9

22. Lewin S, Hendry M, Chandler J, et al. Assessing the complexity of interventions within systematic reviews: development, content and use of a new tool (iCAT_SR). BMC Med Res Methodol. 2017;17(1):1-3.

23. Gardner T, Refshauge K, Smith L, McAuley J, Hübscher M, Goodall S. Physiotherapists' beliefs and attitudes influence clinical practice in chronic low back pain: a systematic review of quantitative and qualitative studies. $J$ Physiother. 2017;63(3):132-143. doi:10.1016/j.jphys.2017.05.017

24. Caneiro JP, Bunzli S, O'Sullivan P. Beliefs about the body and pain: the critical role in musculoskeletal pain management. Braz J Phys Ther. 2021;25(1):17-29. doi:10.1016/j.bjpt.2020.06.003

25. Holopainen R, Simpson P, Piirainen A, et al. Physiotherapists' perceptions of learning and implementing a biopsychosocial intervention to treat musculoskeletal pain conditions: a systematic review and metasynthesis of qualitative studies. Pain. 2020;161(6):1150-1168. doi:10.1097/j.pain.0000000000001809

26. Bryant C, Lewis P, Bennell KL, et al. Can physical therapists deliver a pain coping skills program? An examination of training processes and outcomes. Phys Ther. 2014;94(10):1443-1454. doi:10.2522/ ptj.20130444

27. Godfrey E, Wileman V, Galea Holmes M, et al. Physical therapy informed by Acceptance and Commitment Therapy (PACT) versus usual care physical therapy for adults with chronic low back pain: a randomized controlled trial. J Pain. 2020;21(1):71-81. doi:10.1016/ j.jpain.2019.05.012
28. Simpson P, Holopainen R, Schütze R, et al. Training of physical therapists to deliver individualized biopsychosocial interventions to treat musculoskeletal pain conditions: a scoping review. Phys Ther. 2021;101(10):pzab188. doi:10.1093/ptj/pzab188

29. Mardian AS, Hanson ER, Villarroel L, et al. Flipping the pain care model: a sociopsychobiological approach to high-value chronic pain care. Pain Med. 2020;21(6):1168-1180. doi:10.1093/pm/pnz336

30. Slade SC, Kent P, Patel S, Bucknall T, Buchbinder R. Barriers to primary care clinician adherence to clinical guidelines for the management of low back pain: a systematic review and metasynthesis of qualitative studies. Clin J Pain. 2016;32(9):800-816. doi:10.1097/ AJP.0000000000000324

31. Kilbourne AM, Neumann MS, Pincus HA, Bauer MS, Stall R. Implementing evidence-based interventions in health care: application of the replicating effective programs framework. Implement Sci. 2007;2(1):42-51. doi:10.1186/1748-5908-2-42

32. Kirchner JE, Smith JL, Powell BJ, Waltz TJ, Proctor EK. Getting a clinical innovation into practice: an introduction to implementation strategies. Psychiatry Res. 2020;283:112467. doi:10.1016/j.psychres.2019.06.042

33. Waltz TJ, Powell BJ, Matthieu MM, et al. Consensus on strategies for implementing high priority mental health care practices within the US department of veterans affairs. Implement Res Pract. 2021;2:26334895211004610. doi:10.1177/26334895211004607

34. Brown CH, Curran G, Palinkas LA, et al. An overview of research and evaluation designs for dissemination and implementation. Annu Rev Public Health. 2017;38(1):1-22. doi:10.1146/annurev-publhealth -031816-044215

35. Ballengee LA, Covington JK, George SZ. Introduction of a psychologically informed educational intervention for pre-licensure physical therapists in a classroom setting. BMC Med Educ. 2020;20(1):382. doi:10.1186/s12909-020-02272-5

36. Hoeger Bement MK, St. Marie BJ, Nordstrom TM, et al. An interprofessional consensus of core competencies for prelicensure education in pain management: curriculum application for physical therapy. Phys Ther. 2014;94(4):451-465. doi:10.2522/ptj.20130346

37. CAPTE accreditation handbook. Available from: http://www.capteon line.org/AccreditationHandbook/. Accessed September 29, 2020.

38. Denneny D, Frijdal Nee Klapper A, Bianchi-Berthouze N, et al. The application of psychologically informed practice: observations of experienced physiotherapists working with people with chronic pain. Physiotherapy. 2020;106:163-173. doi:10.1016/j.physio.2019.01.014

39. Bement MKH, Sluka KA. The current state of physical therapy pain curricula in the United States: a faculty survey. J Pain. 2015;16 (2):144-152. doi:10.1016/j.jpain.2014.11.001

40. IASP curriculum outline on pain for physical Therapy. IASP. Available from: https://www.iasp-pain.org/Education/ CurriculumDetail.aspx?ItemNumber=2055. Accessed March 16, 2021.

41. NQF: National Quality Partners ${ }^{\mathrm{TM}}$ Opioid Stewardship action team. Available from: https://www.qualityforum.org/National_Quality_ Partners_Opioid_Stewardship_Action_Team.aspx. Accessed June 17, 2021.

42. Landes SJ, McBain SA, Curran GM. An introduction to effectiveness-implementation hybrid designs. Psychiatry Res. 2019;280:112513. doi:10.1016/j.psychres.2019.112513

43. Curran GM, Bauer M, Mittman B, Pyne JM, Stetler C. Effectivenessimplementation hybrid designs. Med Care. 2012;50(3):217-226. doi:10.1097/MLR.0b013e3182408812

44. Powell BJ, Waltz TJ, Chinman MJ, et al. A refined compilation of implementation strategies: results from the Expert Recommendations for Implementing Change (ERIC) project. Implement Sci. 2015;10 (1):21. doi:10.1186/s13012-015-0209-1

45. Fernandez ME, Ten Hoor GA, van Lieshout S, et al. Implementation mapping: using intervention mapping to develop implementation strategies. Front Public Health. 2019;7:158. doi:10.3389/ fpubh.2019.00158 
46. Beauchemin M, Cohn E, Shelton RC. Implementation of clinical practice guidelines in the healthcare setting: a concept analysis. ANS Adv Nurs Sci. 2019;42(4):307-324. doi:10.1097/ ANS.0000000000000263

47. Middleton A, Fitzgerald GK, Delitto A, Saper RB, Gergen Barnett K, Stevans J. Implementing stratified care for acute low back pain in primary care using the STarT Back instrument: a process evaluation within the context of a large pragmatic cluster randomized trial. BMC Musculoskelet Disord. 2020;21(1):776. doi:10.1186/s12891-020-03800-6

48. Roseen EJ, Conyers FG, Atlas SJ, Mehta DH. Initial management of acute and chronic low back pain: responses from brief interviews of primary care providers. J Altern Complement Med. 2021;27(S1): S106-S114. doi:10.1089/acm.2020.0391

49. Kamper SJ, Logan G, Copsey B, et al. What is usual care for low back pain? A systematic review of health care provided to patients with low back pain in family practice and emergency departments. Pain. 2020;161(4):694-702. doi:10.1097/j.pain.0000000000001751

50. Bonakdar R, Palanker D, Sweeney MM. Analysis of state insurance coverage for nonpharmacologic treatment of low back pain as recommended by the American college of physicians guidelines. Glob Adv Health Med. 2019;8:2164956119855629. doi:10.1177/ 2164956119855629

51. Damschroder LJ, Aron DC, Keith RE, Kirsh SR, Alexander JA, Lowery JC. Fostering implementation of health services research findings into practice: a consolidated framework for advancing implementation science. Implement Sci. 2009;4(1):50. doi:10.1186/1748-5908-4-50

52. Keith RE, Crosson JC, O’Malley AS, Cromp D, Taylor EF. Using the Consolidated Framework for Implementation Research (CFIR) to produce actionable findings: a rapid-cycle evaluation approach to improving implementation. Implement Sci. 2017;12(1):1-12. doi:10.1186/s13012-017-0550-7
53. Cheng L, Leon V, Liang A, et al. Patient-centered care in physical therapy: definition, operationalization, and outcome measures. Phys Ther Rev. 2016;21(2):109-123. doi:10.1080/10833196.2016.1228558

54. Wijma AJ, Bletterman AN, Clark JR, et al. Patient-centeredness in physiotherapy: what does it entail? A systematic review of qualitative studies. Physiother Theory Pract. 2017;33(11):825-840. doi:10.1080/ 09593985.2017.1357151

55. Driver C, Kean B, Oprescu F, Lovell GP. Knowledge, behaviors, attitudes and beliefs of physiotherapists towards the use of psychological interventions in physiotherapy practice: a systematic review. Disabil Rehabil. 2017;39(22):2237-2249. doi:10.1080/ 09638288.2016 .1223176

56. Nielsen M, Keefe FJ, Bennell K, Jull GA. Physical therapistdelivered cognitive-behavioral therapy: a qualitative study of physical therapists' perceptions and experiences. Phys Ther. 2014;94 (2):197-209. doi:10.2522/ptj.20130047

57. Force APCT. Addressing obstacles to evidence-informed pain care. AMA J Ethics. 2020;22(8):709-717. doi:10.1001/amajethics.2020.709.

58. Institute of Medicine (US) Committee on Advancing Pain Research C. Care of people with pain. National Academies Press (US); 2011. Available from: http:/www.ncbi.nlm.nih.gov/books/NBK92517/. Accessed July 27, 2021.

59. Beneciuk JM, George SZ, Greco CM, et al. Targeted interventions to prevent transitioning from acute to chronic low back pain in high-risk patients: development and delivery of a pragmatic training course of psychologically informed physical therapy for the TARGET trial. Trials. 2019;20(1):256. doi:10.1186/s13063-019-3350-3
Journal of Pain Research

\section{Publish your work in this journal}

The Journal of Pain Research is an international, peer reviewed, open access, online journal that welcomes laboratory and clinical findings in the fields of pain research and the prevention and management of pain. Original research, reviews, symposium reports, hypothesis formation and commentaries are all considered for publication. The manuscript management system is completely online and includes a very quick and fair peer-review system, which is all easy to use. Visit http:// www.dovepress.com/testimonials.php to read real quotes from published authors. 